\title{
ELABORATION AND CHARACTERIZATION OF A NATURAL COMPOSITE MATERIAL BASED ON COLLOIDAL PARTICLES OF MICROCRYSTALLINE CELLULOSE COATED WITH MODIFIED STARCH
}

\author{
IMENE BOULHAIA,,$* * *$ NADJI MOULAI-MOSTEFA, ${ }^{*}$ ABDELKADER HADJSADOK ${ }^{* *}$ \\ and ALI AOUABED** \\ *LME, University of Medea, Ain D'Heb, 26001 Medea, Algeria \\ ** LAFPC, University of Blida 1, Route de Soumaa, 09000 Blida, Algeria \\ Corresponding author: Imene Boulhaia, imeneboulhaia@yahoo.fr
}

Received November 26, 2019

Physicochemical and rheological analyses were realized on microcrystalline cellulose (MCC) of colloidal grade, coated with octenyl succinic anhydride (OSA) starch. The objective was to prepare a biobased material (OSA-Cel), with new characteristics relating to both surface activity and viscosity. The chemical structures of MCC, OSA-starch and OSACel were studied using FT-IR and XRD analyses, and their surface aspects were determined by surface tension measurements. From the results obtained, it was noticed that the FT-IR spectra showed great similarity between the characteristics of the crude MCC and those of OSA-Cel. Analysis by XRD showed a peak that was attributed to the crystalline phase of the cellulose. Furthermore, it was shown that OSA-starch interacts notably with MCC. The rheological properties at different concentrations of OSA-Cel showed the same behavior as that of MCC, a three-region viscosity profile (thinning, shear thickening and an infinite shear plateau).

Keywords: microcrystalline cellulose, OSA-starch, composites, surface tension, rheology

\section{INTRODUCTION}

The use of nanomaterials as reinforcing phase in polymeric matrices to form nanocomposites has attracted much research attention due to their ability to improve the mechanical properties of such systems. ${ }^{1}$ Hence, biobased materials have been a subject of immense research interest in recent years due to their great potential to produce a variety of biocompatible products. ${ }^{2-4}$

Microcrystalline cellulose (MCC) is a pure cellulose that has been partially depolymerized via hydrolysis in hydrochloric acid. ${ }^{5}$ The process helps to remove the disordered regions of the cellulose, only the highly ordered regions remaining. ${ }^{6}$ Its crystalline nature and involvement in hydrogen bonding make significant contributions to the chemical and physical properties of MCC, in a variety of environments. ${ }^{7}$ Furthermore, the development of colloidal MCC made available much finer particle forms of highly purified crystalline cellulose, and more importantly, aqueous suspensions. ${ }^{3}$

MCC is utilized in the pharmaceutical industry due to its interactive behavior and biodegradabi- lity $;^{5}$ it is widely used for the stabilization of various beverages. ${ }^{8}$ In the food industry, MCC is employed due to its heat stability, thickening ability and flow control. ${ }^{9}$ However, the hydrophilic nature of cellulose and its tendency to agglomerate are major problems that prevent its uniform dispersion in solutions. ${ }^{10}$ To avoid these limitations, surface modification has been proposed for better dispersion of cellulose fibers in a composite matrix. ${ }^{2,11}$ In addition, surface functionalization was used to reduce the aggregation of cellulose at the microscopic scale in hydrophobic matrices. ${ }^{12,13}$ As regards colloidal suspensions, a common practice to avoid altering their stability and rheological properties is the addition of polymers to suspensions. However, the presence of two polymers in the same solution can generate interactions, ${ }^{14}$ they can cause two types of steric interactions among particles (either by polymer adsorption onto particle surfaces or by depletion by non-adsorbing polymers). Both of them can lead to flocculation and the formation of gels. ${ }^{15}$ 
Redispersible MCC products containing carboxymethyl cellulose (CMC) as dispersing aid are commercially available. ${ }^{16}$ Furthermore, coating cellulose microfibrils with anionic polymeric additives, such as CMC and polyacrylate, has been found to form a water soluble interfacial film around the cellulose microfibrils while drying, which allows the recovering of rheological properties after redispersion of dried samples. ${ }^{16-18}$

In this study, a suitable approach has been developed to prevent agglomeration of MCC during dispersion by partially or completely covering MCC with octenyl succinic anhydride (OSA) modified starch. This strategy is based on a simple coating technique involving a modest physical treatment, which is convenient and easily available. $^{11}$

OSA-starch is a corn starch modified by an esterification reaction between the hydroxyl groups of starch and the octenyl succinic anhydride groups. ${ }^{19}$ Furthermore, the use of OSAstarch is authorized by the food and drug administration. $^{20}$ Its modification with hydrophobic groups gives the starch amphiphilic properties. ${ }^{21}$

The objectives of this work were to develop a new material based on MCC, in the form of colloidal suspension, obtained by hydrolysis with sulfuric acid. The second phase consists in coating the colloidal particles obtained with OSAstarch in order to prepare a bio-based composite material (OSA-Cel), which can be used as stabilizer in the formulation of dispersed systems or as reinforcing filler in a polymeric matrix. The obtained product was characterized in terms of chemical structure, morphology, surface aspects and rheology.

\section{EXPERIMENTAL \\ Materials}

Octenyl succinic anhydride (OSA) modified starch was obtained from National Starch and Chemicals (GmbH, Germany). Microcrystalline cellulose (MCC, Avicel PH 101) was commercially obtained. Sodium azide, sulfuric acid and sodium hydroxide were purchased from Sigma Aldrich (Switzerland).

\section{Preparation of colloidal MCC}

The preparation of MCC in the form of colloidal particles was achieved according to the method proposed by Hasani et $\mathrm{al}^{22}$ with some modifications. This protocol consists of a first step of hydrolysis with sulfuric acid, followed by several washing steps, dialysis and a desulfation step. Hydrolysis was performed using a volume of $1000 \mathrm{~mL}$ of sulfuric acid solution (64 wt\%). The reaction being very exothermic, the solution was introduced into a flask, which was placed in a thermostatic bath to reach a temperature of $45{ }^{\circ} \mathrm{C}$. Once the temperature was stabilized, a quantity of $50 \mathrm{~g}$ of MCC was introduced into the acidic solution. After 30 min of hydrolysis, the suspension was centrifuged for $10 \mathrm{~min}$ at $11000 \mathrm{rpm}$ in order to remove the largest amount of acid. The pellet obtained was redispersed in distilled water and then centrifuged again at 11,000 rpm for $10 \mathrm{~min}$ using a 330K Sigma centrifuge ( $\mathrm{GmbH}$, Germany). This step was repeated until the $\mathrm{pH}$ of the supernatant liquid was close to the $\mathrm{pH}$ of distilled water. The microcrystals constituting the final pellet were dispersed in distilled water. For the removal of traces of acid, the suspension was then placed in a dialysis membrane and dialyzed until the conductivity of the dialysis water was stable (substantially equal to that of the added distilled water). This operation lasted 4 to 5 days and it was necessary to change the bath of distilled water three times a day. The desulfation operation was carried out with a volume of $500 \mathrm{~mL}$ of a suspension of MCC ( 9 wt\%) mixed with a solution of $\mathrm{NaOH}(2 \mathrm{M})$. The mixture was stirred at $65{ }^{\circ} \mathrm{C}$ for $5 \mathrm{~h}$; the desulfation process was followed by several washing steps to remove excess salts and a dialysis step. ${ }^{23}$

\section{Elaboration of OSA-Cel}

The quantity of the colloidal MCC suspension to be coated with OSA-starch was determined gravimetrically. ${ }^{16} \mathrm{~A}$ well-defined volume of MCC was utilized and the suspension was left in the oven at 40 ${ }^{\circ} \mathrm{C}$, until it dried completely. The dry matter was weighed and the suspension concentration was determined, corresponding to the volume utilized in \% (in wt). The colloidal suspension (100 g) was mixed with OSA starch $(10 \mathrm{~g})$. The mixture was placed in a bath at $40{ }^{\circ} \mathrm{C}$ under stirring for $10 \mathrm{~min}$ until the total dispersion of OSA starch particles in MCC slurry was achieved. The obtained product (OSA-Cel) was then dried in the oven at $40{ }^{\circ} \mathrm{C}$ until it was completely dry.

\section{Characterization methods}

The chemical structures of MCC, OSA-starch and OSA-Cel were qualitatively analyzed using FT-IR spectroscopy (Bruker Optics, Inc., Tensor 27, Germany). Samples were prepared by grinding the fine powder with $\mathrm{KBr}$, and scanned over a wavenumber range from 400 to $4000 \mathrm{~cm}^{-1}$.

X-ray diffraction (XRD) was performed in the reflection mode, using a Philips PW 1800 apparatus (copper $\mathrm{K} \alpha$ line $\lambda=1.5418 \AA$ ). The spectra of the different samples were recorded in a $2 \theta$ domain between $15^{\circ}$ and $70^{\circ}$, with an angular increment of 0.04 . The crystallinity index (CI) was determined from the XRD spectra, according to the method reported by Buschle-Diller and Zeronian, ${ }^{24}$ using Equation 1:

$$
\mathrm{CI}=1-\left(\mathrm{I}_{\min } / \mathrm{I}_{\max }\right)
$$


where $I_{\min }$ is the intensity minimum between $2 \theta=18$ and $19^{\circ}$, and $\mathrm{I}_{\max }$ is the intensity of the crystalline peak at the maximum between $2 \theta=22$ and $23^{\circ}$.

The surface tensions of the dispersions were measured using a bi-directional tensiometer of $\mathrm{Du}$ Noüy model 70545 .

\section{Rheological analysis}

The rheological measurements were performed with a constraint and strain-strain rheometer (Anton Paar, Physica MCR 302, Germany), using a plate-plate geometry (diameter of $25 \mathrm{~mm}$ ). The gap used was 1 $\mathrm{mm}$, which means that the possible interface effects were very small. The measurement temperature was $20 \pm 0.1{ }^{\circ} \mathrm{C}$; it was stabilized in less than $1 \mathrm{~min}$. For the flow tests, shear viscosity was measured at a shear rate ranging from $10^{-3}$ to $10^{3} \mathrm{~s}^{-1}$. The rheological tests were performed for the cellulose suspension (MCC) at a concentration of $0.3 \mathrm{wt} \%$. The effects of OSA-Cel on the viscosity curves were also tested at concentrations ranging between 1.5 and $10 \mathrm{wt} \%$.

\section{Determination of morphology and size distribution}

The morphological characterization was performed with an optical microscope (Optika DM-25). The purpose of this characterization was the evaluation of particle dispersion in the continuous phase (enlargement of 40x). The size distribution was evaluated using a combination of light microscopy and ImageJ software. ${ }^{24}$ The first step was to introduce into the ImageJ software the scale associating the actual micrometric size of particles with the size of the microscopic photo in pixels. For this, a micrometric graduated blade, with $10 \mu \mathrm{m}$ unit and a length of 0.5 $\mathrm{cm}$, was used. The second step was to load the microscopic photo by the ImageJ software and transform it into 8 bit format, it was then contrasted to allow physical separation in the continuous phase of the dispersed particles, which were simulated as ellipses in order to calculate their number and surface. The surfaces of the ellipses were assumed to be circular in shape, and thus the size $\left(D_{i}\right)$ of the particles equivalent to the full circular shape was determined $\left(S_{i}\right.$ $=\pi D_{i}^{2} / 4, i$ represents the number associated with the particles). Finally, a numerical table was obtained using STATISTICA software, in which the number of particles detected and their size (in $\mu \mathrm{m}$ ) were summarized. The statistical distribution of the particle frequency, expressed as percentage, versus size is illustrated by a histogram. In parallel, the percentage of cumulative frequencies was determined, from which we highlight the three characteristic parameters $\left(D_{10}\right.$, $D_{50}$ and $D_{90}$ ).

\section{Statistical analysis}

The statistically significant differences between the means were evaluated by performing the data analyses in triplicate. The results were expressed as mean \pm standard deviation (SD).

\section{RESULTS AND DISCUSSION Chemical structure of cellulose and its derivatives}

Figure 1 presents the FT-IR spectra of colloidal MCC after desulfation, OSA-Cel and OSA-starch. From the spectra obtained, it may be noted that the colloidal MCC spectrum contains the vibration bands of free or bound $-\mathrm{OH}$ bond (hydrogen bond) at $3334.56 \mathrm{~cm}^{-1}$, and the vibration of the $\mathrm{CH}$ and $\mathrm{CH}_{2}$ bond at $2901.65 \mathrm{~cm}^{-}$ 1. The peaks between 1000 and $1080 \mathrm{~cm}^{-1}$ correspond to the vibrations of the $\mathrm{COH}$ bond of a primary alcohol and $\mathrm{C}=\mathrm{O}$ of an ester. After the operation of coating, the cellulose band partially overlaps with that of OSA-starch, but is still well distinguished. This proves that the thickness of the cellulose fiber coating with OSA-starch is greater than the penetration depth of the IR radiation in the sample (a few $\mu \mathrm{m}$ ). The degree of substitution of OSA-starch is low, of the order of $0.02{ }^{25}$ This does not allow the appearance of additional peaks of starch by the modification at 1723 and $1563 \mathrm{~cm}^{-1}$ of an ester.

The X-ray diffraction patterns (XRD) of the three studied materials are illustrated in Figure 2. The diffractogram analysis reveals five intense diffraction peaks at $15.13^{\circ}, 20.62^{\circ}, 22.17^{\circ}, 34.01^{\circ}$ and $18.69^{\circ}$ for OSA-starch. For MCC, the first four peaks are attributed to the crystalline phase of MCC. The peak intensities of OSA-Cel are lower than those of MCC, but the peak width of both samples is the same. The comparison of the XRD diffractograms of initial MCC and that after size and surface modification (OSA-Cel) makes it possible to highlight this modification. Regarding OSA-starch, the XRD spectrum shows a low degree of crystallinity with the DS compared to that of native starch, which shows a morphological crystallinity typical of a type of double helix structure. $^{26,27}$ The obtained crystallinity index (CI) values were found equal to 0.76 for MCC and 0.68 for OSA-Cel. These results are in agreement with those obtained by Ioelovich ${ }^{28}$ who found CI values ranging between 0.72 and 0.75 for MCC samples. 


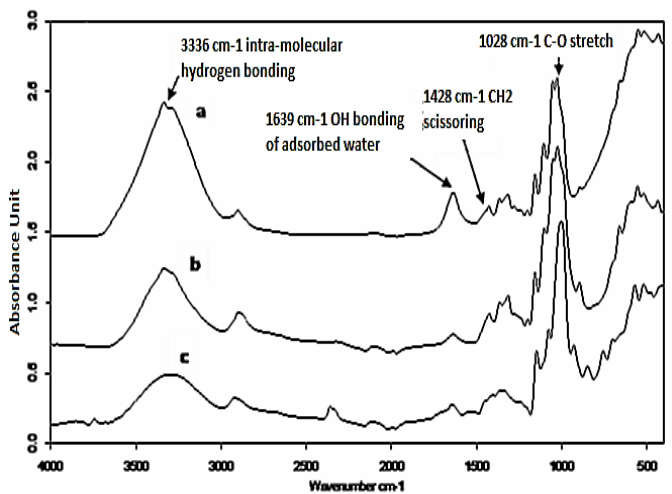

Figure 1: FTIR spectra of (a) OSA-Cel, (b) MCC after desulfation (colloidal MCC), and (c)

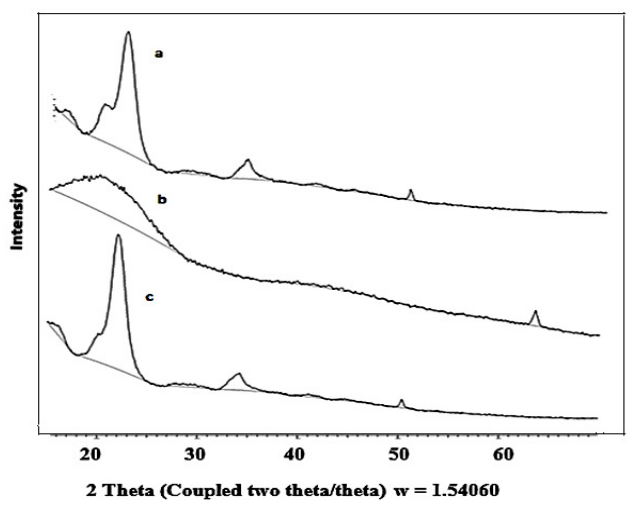

Figure 2: X-ray diffraction patterns of (a) OSACel, (b) OSA-starch and (c) MCC OSA-starch

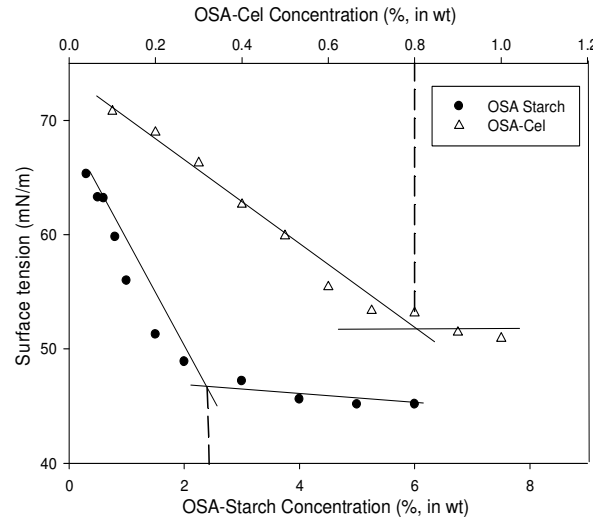

Figure 3: Surface tension $v s$ concentration of OSA-Cel and OSA-starch

\section{Surface properties}

The surface aspects of OSA-starch and OSACel were analyzed by surface tension measurements. The values of surface tension were found to be lower for OSA-starch, which confirms its surfactant character. Some authors, such as Onesippe and Lagerge, ${ }^{29}$ reported the effect of adding polyelectrolytes on the decrease in surface tension. However, the results obtained demonstrated an opposite effect. The addition of MCC increases the surface tension due to desulfation and removal of anionic groups. Previous works ${ }^{30,31}$ showed that cellulose has no noticeable effect on surface activity, which explains the increase in surface tension when MCC is added. However, the values of the critical aggregation concentration (cac) measured were, respectively, $2.4 \%$ for OSA-starch and $0.8 \%$ for OSA-Cel. This is due to the coating of colloidal MCC with the modified starch, which brings the polymer chains of OSA-starch closer and therefore lowers the cac value.

The obtained results revealed large differences in the behavior of MCC in the presence of OSAstarch acting as surfactant. It was demonstrated, on the one hand, that cellulose is responsible for surfactant desorption from the interface and, on the other hand, the surfactant interacts significantly with the polymer. This was attributed to the strong interactions between the surfactant micelles and the polymer particles. ${ }^{32}$

\section{Morphology and particle size distribution}

Figure 4 shows microscopic images (with magnification of $\mathrm{x} 40$ ) of MCC before and after hydrolysis and desulfation, as well as that of the product developed. From the observed images, it may be noted that initial MCC has very large particles in comparison with those of MCC after hydrolysis, which confirms the hypothesis of acid treatment. 

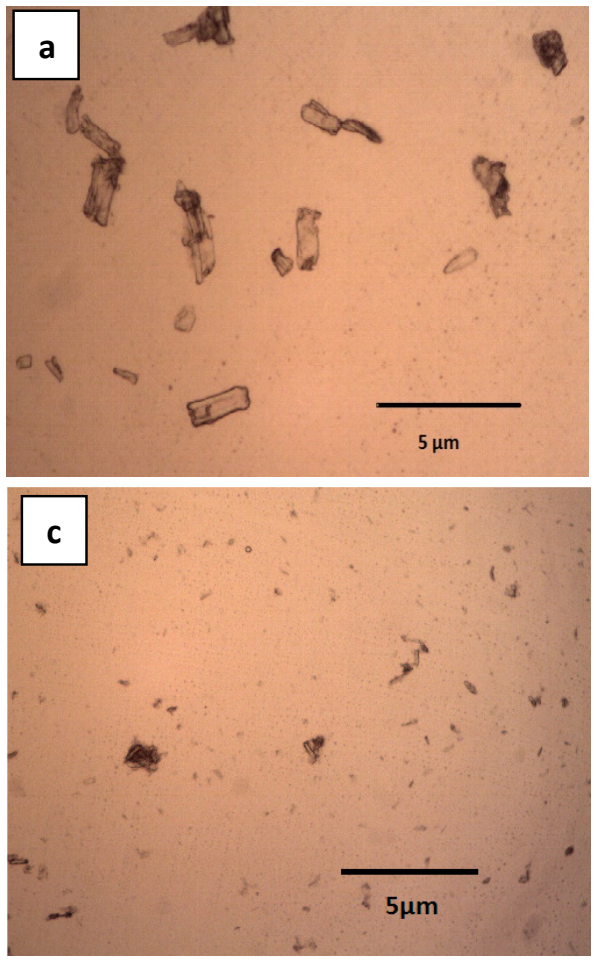
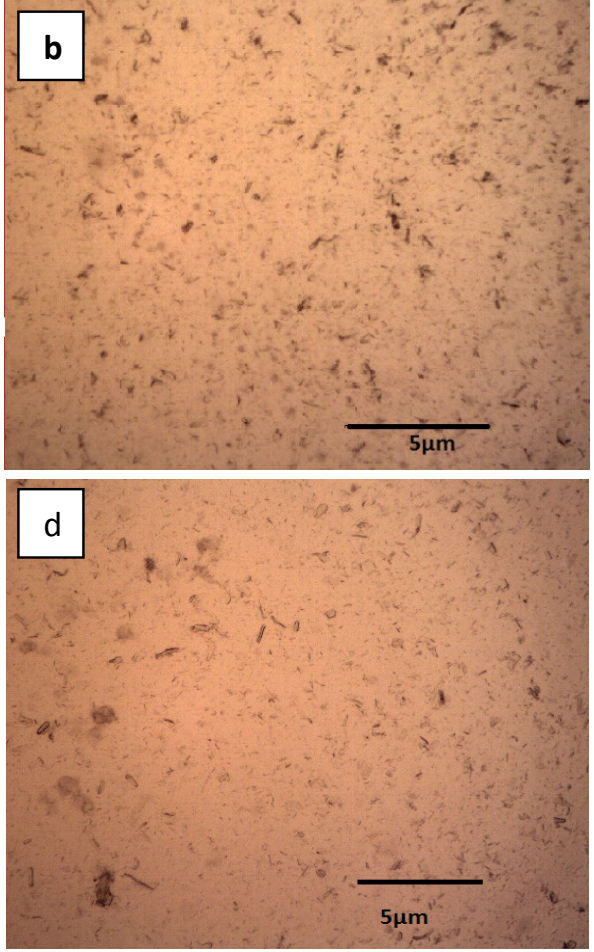

Figure 4: Microscopic images of raw MCC (a), MCC after hydrolysis (b), MCC after desulfation (c) and OSA-Cel suspension (d)

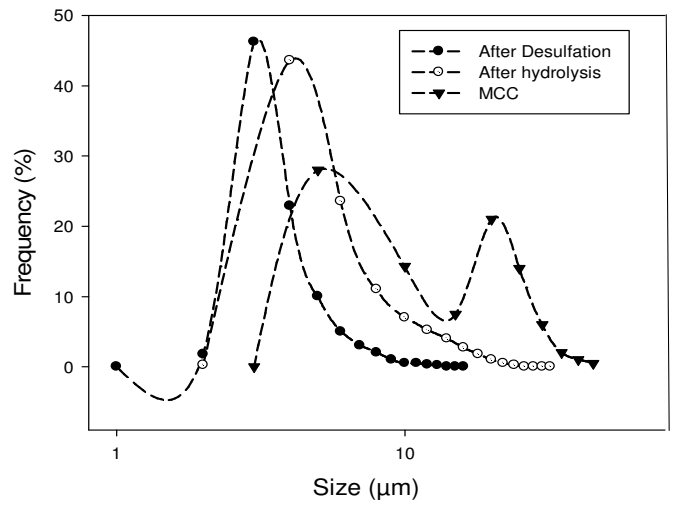

Figure 5: Cumulative frequency curves of different samples of MCC

Figure 5 illustrates the particle size distribution of the raw MCC in suspension. The powder was not subjected to any chemical or physicochemical treatment. The profile of this distribution seems to be of bimodal type, with a first mode around 2.5 $\mu \mathrm{m}$, which represents $80 \%$ of the population, and a second mode, representing a small population, which is close to the value of $17 \mu \mathrm{m}$. It was also noticed from the cumulative frequency curve that $D_{10}$ and $D_{50}$ are not present. On the other hand, the value of $D_{90}$ is equal to $13.5 \mu \mathrm{m}$. In other words, $90 \%$ of the population has a diameter less than $13.5 \mu \mathrm{m}$, which implies that the grade of this ingredient is micronized, but not colloidal.

Concerning MCC after hydrolysis and after desulfation, it is clear that the distribution is monomodal, but it is more extensive next to the large particles. The average diameter value of this distribution is equal to $3.75 \mu \mathrm{m}$; it fluctuates, in a confidence interval defined at $95 \%$, between 0.76 
and $5.7 \mu \mathrm{m}$. The extreme particle size values that are exceptionally present are below $0.1 \mu \mathrm{m}$ and above $7.6 \mu \mathrm{m}$. From the cumulative frequency curve, the following values are deduced: $D_{10}=2.2$ $\mu \mathrm{m}, D_{50}=3 \mu \mathrm{m}$ and $D_{90}=6.5 \mu \mathrm{m}$. These results

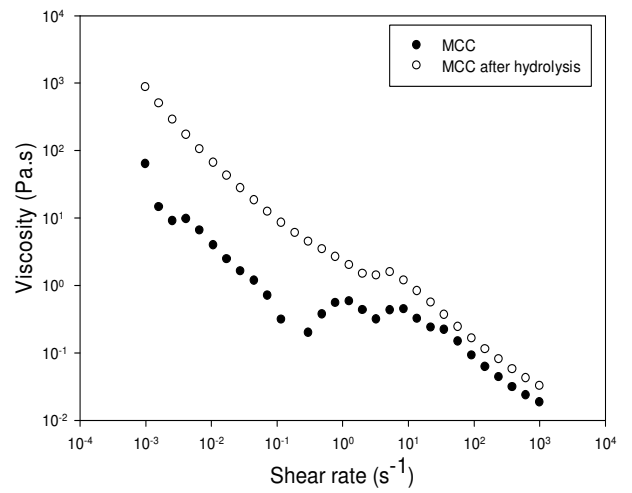

Figure 6: Viscosity variation of raw MCC and hydrolyzed MCC versus shear rate at a concentration of $3 \mathrm{wt} \%$

\section{Rheological properties}

The flow curves expressing the variation of the viscosity as a function of the shear rate of a colloidal suspension of MCC ( $3 \mathrm{wt} \%$ ) before and after hydrolysis are illustrated in Figure 6. It may be noticed that the rheological behavior is significantly influenced by the physiochemical properties of the particles. Three well-defined regions are observed. At a low shear rate, the viscosity is high and then decreases with the shear rate. This shear-thinning behavior shows the alignment of the cellulose rods under flow. At an intermediate shear rate $\left(1-10 \mathrm{~s}^{-1}\right)$, the decrease in viscosity is lower. This is due to a high level of order of the cellulose rods because of the interactions between the particles. However, at higher shear rates $\left(>10 \mathrm{~s}^{-1}\right)$, this ordered structure decomposes and the rods become individualized. The rheological behavior is typical of liquid crystalline polymers. Ureña-Benavides et al. ${ }^{33}$ reported that the rheological properties and phase behavior of such materials are mainly related to concentration and temperature. It has also been observed that the viscosity of raw MCC after Ultra-Turrax grinding is much lower than that of MCC after hydrolysis.

In Figure 7, the flow curves of OSA-Cel at different concentrations are presented. It was noticed that the rheological behavior of OSA-Cel is identical to that of colloidal MCC. These two materials exhibit a shear-thinning behavior. These findings are in agreement with those found by showed that the majority of the population has a diameter of less than $6.5 \mu \mathrm{m}$. Thus, at the end of the desulfation process of MCC, a powder with a micronized grade (size $<10 \mu \mathrm{m}$ ) is obtained.

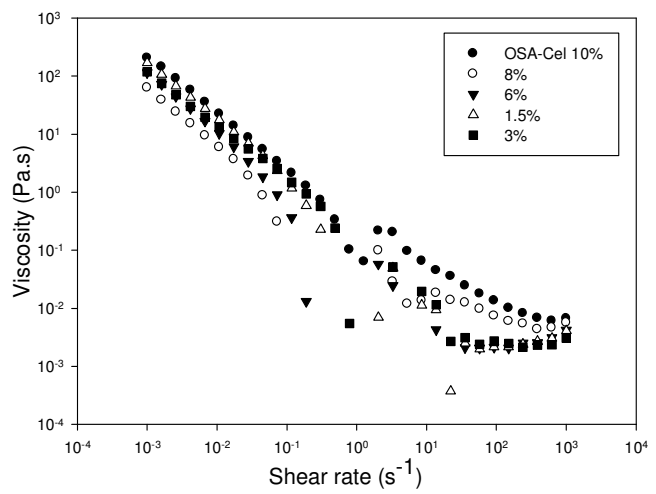

Figure 7: Flow curves of OSA-Cel suspensions at different concentrations versus shear rate

Shafiei-Sabet et $a l .{ }^{34}$ In addition, the flow curves in both cases have three regions. In region I, with a very low shear rate, a shear-thinning behavior is observed in the absence of a Newtonian plateau. In the second region (at intermediate shear rates), a short pseudo-Newtonian plateau is observed, followed by the third region, again describing the manifestation of shear-thinning behavior.

These results can be explained in two ways. The first possible explanation is the presence of two different populations of MCC and OSA-Cel particles of average size and less aggregated than the second population formed by the residual fractions of MCC particles, where region I is attributed to the flow of smaller particles and, regions II and III correspond to the flow of MCC particles. The second hypothesis is the presence of weak intermolecular compounds and intramolecular hydrophobic associations due to the presence of $-\mathrm{CH}_{2^{-}}$on the carboxymethyl group $\left(-\mathrm{CH}_{2}-\mathrm{COO}^{-}\right)$. The first region describes the flow resulting from the phenomenon of destructuration initiated by the breaking of intermolecular bonds, where the predominant mode of interaction is intramolecular, ${ }^{35}$ while the second region corresponds to the rupture of intramolecular bonds with some resistance, expressed here by the presence of the transition equilibrium plateau. 


\section{CONCLUSION}

The main purpose of this investigation was to explore new raw materials that are readily available, at a relatively low cost. Microcrystalline cellulose (MCC) and OSAstarch were selected, as they possess interesting functional properties. The idea was to develop a combination of MCC of colloidal grade with OSA-starch to produce a composite material (OSA-Cel) that can be used as suspending agent in the field of dispersed systems.

Colloidal MCC was prepared by chemical treatment of the raw MCC. The particle distribution of the obtained suspension was found to be of the unimodal type, where the majority of the population had a diameter of $6 \mu \mathrm{m}$. It was also found that the product developed (OSA-Cel) has a surfactant power thanks to its composition including OSA-starch. The rheological analysis revealed the formation of three-dimensional networks. From the flow curves, the existence of different populations of particles was noticed, which are responsible for the different behaviors observed.

In conclusion, the product developed seems to have better characteristics from the point of view of particle size, stability, as well as rheological and surface properties, in addition to its very economical production.

\section{REFERENCES}

1 X. Yao, X. Qi, Y. He, D. Tan, F. Chen et al., ACS Appl. Mater. Inter., 6, 2497 (2014), https://doi.org/10.1021/am4056694

2 H. M. Ahsan, X. Zhang, Y. Li, B. Li and S. Liu, Int. J. Biol. Macromol., 132, $1176 \quad$ (2019), https://doi.org/10.1016/j.ijbiomac.2019.04.051

D. Trache, M. H. Hussin, C. T. Hui Chuin, S. Sabar, M. R. N. Fazita et al., Int. J. Biol. Macromol., 93, 789 (2016), https://doi.org/10.1016/j.ijbiomac.2016.09.056

4 C. W. Miao and W. Y. Hamad, Cellulose, 20, 2221 (2013), https://doi.org/10.1007/s10570-013-0007-3

S. S. Z. Hindi, Nanosci. Nanotechnol. Res., 4, 17 (2017), https://doi.org/10.12691/nnr-4-1-3

6 A. Tayeb, E. Amini, S. Ghasemi and M. Tajvidi, Molecules, 23, $2684 \quad$ (2018), https://doi.org/10.3390/molecules23102684

7 H. T. Sahin and M. B. Arslan, Int. J. Mol. Sci., 9, 78 (2008), https://doi.org/10.3390/ijms9010078

8 K. Yaginuma and T. Kijima, J. Disp. Sci. Technol., 27 , 941

(2006), https://doi.org/10.1080/01932690600766306

9 J. Nsor-Atindana, M. Chen, H. D. Goff, F. Zhong, H. R. Sharif et al., Carbohyd. Polym., 172, 159 (2017), https://doi.org/10.1016/j.carbpol.2017.04.021
10 D. J. Gardner, G. S. Oporto, R. Mills and M. A. S. A. Samir, J. Adhes. Sci. Technol., 22, 545 (2008), https://doi.org/10.1163/156856108X295509

11 Y. Pan, Y. Liu, Q. Cheng, Y. Celikbag, B. K. Via et al., Eur. J. Wood Wood Prod., 74, 763 (2016), https://doi.org/10.1007/s00107-016-1030-5

12 A. J. De Menezes, G. Siqueira, A. A. Curvelo and A. Dufresne, Polymer, 50, 4552 (2009), https://doi.org/10.1016/j.polymer.2009.07.038

${ }_{13}$ A. L. Goffin, J. M. Raquez, E. Duquesne, G. Siqueira, Y. Habibi et al., Biomacromolecules, 12, 2456 (2011), https://doi.org/10.1021/bm200581h

${ }_{14}$ G. J. Fleer, Adv. Colloid Interface Sci., 159, 99 (2010), https://doi.org/10.1016/j.cis.2010.04.004

15 P. Jenkins and M. Snowden, Adv. Colloid Interface Sci., 68, 57 (1996), https://doi.org/10.1016/S00018686(96)90046-9

16 N. Butchosa and Q. Zhou, Cellulose, 21, 4349 (2014), https://doi.org/10.1007/s10570-014-0452-7

17 M. P. Lowys, J. Desbrieres and M. Rinaudo, Food Hydrocoll., $\quad \mathbf{1 5}, \quad 25 \quad$ (2001), https://doi.org/10.1016/S0268-005X(00)00046-1

18 P. Myllytie, S. Holappa, J. Paltakari and J. Laine, Nordic Pulp Pap. Res. J., 24, 125 (2009), https://doi.org/10.3183/NPPRJ-2009-24-02-p125-134

19 M. C. Sweedman, M. J. Tizzotti, C. Schäfer and R. G. Gilbert, Carbohyd. Polym., 92, 905 (2013), https://doi.org/10.1016/j.carbpol.2012.09.040

20 JECFA Reports, "Evaluation of Certain Food Additives", in "Seventy-Ninth Report of the Joint FAO/WHO Expert Committee on Food Additives", WHO Technical Report Series (no. 990), World Health Organization (2014), https://apps.who.int/iris/bitstream/handle/10665/15088 3/9789241209908_eng.pdf?sequence $=1$

21 V. Krstonošić, L. Dokić and J. Milanović, Food Hydrocoll., $\quad \mathbf{2 5}, \quad 361 \quad$ (2011), https://doi.org/10.1016/j.foodhyd.2010.06.014

22 M. Hasani, E. D. Cranston, G. Westman and D. G. Gray, Soft Matter, 4, $2238 \quad$ (2008), https://doi.org/10.1039/B806789A

23 S. Park, J. O. Baker, M. E. Himmel, P. A. Parilla and D. K. Johnson, Biotechnol. Biofuels, 3, 10 (2010), https://doi.org/10.1186/1754-6834-3-10

24 D. Segets, J. M. Lucas, R. N. Klupp Taylor, M. Scheele, H. Zheng et al., ACS Nano, 6, 9021 (2012), https://doi.org/10.1021/nn303130d

25 K. Ezzeroug, N. Moulai-Mostefa and A. Hadjsadok, J. Food Sci. Technol., 55, 4485 (2018), https://doi.org/10.1007/s13197-018-3377-6

26 R. F. Tester, J. Karkalas and X. Qi, J. Cereal Sci., 39 151

(2004),

https://doi.org/10.1016/j.jcs.2003.12.001

${ }_{27}$ S. X. Xie, Q. Liu and S. W. Cui, in "Food Carbohydrates", edited by S.W. Cui, CRC Press, Boca Raton, Florida, 2005, pp. 357-405

28 M. Ioelovich, ChemXpress, 93, 245 (2016), https://www.tsijournals.com/journals/chemxpress.html 


\section{IMENE BOULHAIA et al.}

29 C. Onesippe and S. Lagerge, Colloid Surface. A, 317, 100 (2008), https://doi.org/10.1016/j.colsurfa.2007.10.002

30 J. Tang, M. F. X. Lee, W. Zhang, B. Zhao, R. M. Berry et al., Biomacromolecules, 15, 3052 (2014), https://doi.org/10.1021/bm500663w

31 S. Hiranphinyophat, Y. Asaumi, S. Fujii and Y. Iwasaki, Langmuir, 35, $11443 \quad$ (2019), https://doi.org/10.1021/acs.langmuir.9b01584

32 K. D. Berglund, T. M. Przybycien and R. D. Tilton, Langmuir,

19, 2714

(2003),
33 E. E. Ureña-Benavides, G. Ao, V. A. Davis and C. L. Kitchens, Macromolecules, 44, 8990 (2011), https://doi.org/10.1021/ma201649f

34 S. Shafiei-Sabet, W. Y. Hamad and S. G. Hatzikiriakos, Langmuir, 28, 17124 (2012), https://doi.org/10.1021/la303380v

35 M. M. Yahoum, N. Moulai-Mostefa and D. LeCerf, Carbohyd. Polym., 154, $267 \quad$ (2016), https://doi.org/10.1016/j.carbpol.2016.06.080

https://doi.org/10.1021/la026430f 